\title{
ESTUDOS DE ADSORÇÃO DE POLIAROMÁTICOS SOBRE MCM-41: ESTIMATIVA DA DIFUSIVIDADE SUPERFICIAL EFETIVA
}

\author{
F. M. T. LUNA ${ }^{1}$, D. G. de OLIVEIRA ${ }^{1}$ e C. CAVALCANTE Jr. ${ }^{1}$ \\ ${ }^{1}$ Universidade Federal do Ceará, Departamento de Engenharia Química \\ Grupo de Pesquisas em Separações por Adsorção \\ E-mail para contato: murilo@gpsa.ufc.br
}

\begin{abstract}
RESUMO - Os compostos aromáticos estão presentes em diversas correntes de hidrocarbonetos e sua concentração é diretamente dependente das características do petróleo e dos processamentos ao qual a matéria-prima foi submetida. Várias aplicações industriais utilizam essas correntes como insumos ou solventes, dependendo do tipo e da concentração dos compostos aromáticos. Dentre esses compostos, especificamente os hidrocarbonetos policíclicos aromáticos (HPAs) são conhecidos contaminantes ambientais e têm o potencial carcinogênico e mutagênico comprovado. O principal objetivo deste trabalho foi estudar o processo de adsorção de poliaromáticos em MCM-41 e estimar a difusividade superficial em diferentes condições de concentração de alimentação. Experimentos em leito fixo foram realizados em diferentes concentrações para estudar a dinâmica de adsorção e a modelagem do processo foi realizada utilizando o software gPROMS para estimativa da difusividade superficial efetiva utilizando os dados experimentais.
\end{abstract}

\section{INTRODUÇÃO}

Os hidrocarbonetos poliaromáticos (HPA) são compostos considerados altamente tóxicos, e suas características físico-químicas desfavorecem o processo de biodegradação. Devido ao caráter recalcitrante e aos efeitos nocivos à saúde humana, a Agência de Proteção Ambiental (Environmental Protectin Agency - EPA) nos EUA classificou 17 dos HPA como poluentes prioritários. Além de sua ocorrência natural, os HPA estão presentes como produtos indesejados em atividaddes humanas, como a geração de energia pela queima de combustíveis fósseis e a incineração de lixo. Processos industriais, como a siderurgia, também estão inclusos como fontes desses compostos. Águas superficiais e solos são contaminados pela falta de tratamento e despejo inadequado de correntes industriais e pela deficiência das estruturas de armazenamento nos aterros sanitários (ATSDR 1995; Wang et al., 2007).

Em virtude disso, diferentes tipos de pesquisa estão sendo desenvolvidas com o objetivo de remover HPA de emissões gasosas (Mastral et al. 2002), solos contaminados (Gan et al. 2009) e de correntes industriais e de solventes (Luna et al. 2008). O processo de adsorção se mostra como 


\section{9 a 22 de outubro de 2014 \\ Florianópolis/SC}

um dos mais vantajosos entre os processos analisados por sua baixa demanda energética, pela variedade de adsorventes e pela capacidade de regenerá-los (Ania et al. 2007).

Os materiais do tipo MCM-41 são estruturas mesoporosas cujas paredes são formadas de sílica amorfa e é ordenado de modo a produzir longos canais de poros uniformes. Muito já foi realizado no sentido de entender o potencial adsortivo da estrutura mesoporosa de MCM-41 em diferentes contextos, como a remoção de metais e corantes básicos de soluções aquosas, a adsorção de compostos orgânicos voláteis (Northcotta et al., 2010; Juang et al., 2006; Puanngama e Unob 2008). Estudos comparativos sobre a performance adsortiva de MCM-41 e suas variações dopadas em relação à adsorção de HPA foram apresentadas recentemente (Araújo et al. 2008). Trabalhos anteriores já haviam reportado a adsorção de compostos monoaromáticos no silicato (Ghiaci et al., 2004). Contudo, uma melhor compreensão dos mecanismos difusionais no silicato e do comportamento dos parâmetros de transferência de massa sob diferentes condições de processo ainda se faz necessário.

Este trabalho teve como objetivo o estudo das características cinéticas do processo de remoção de um poliaromático padrão (pireno) em um meio poroso formado por MCM-41. A remoção de pireno de soluções de iso-octano a diferentes concentrações por adsorção foram realizadas em um sistema de leito fixo com o intuito de gerar curvas de ruptura. Os resultados experimentais foram interpretados à perspectiva de um modelo geral de transferência de massa, $o$ modelo de dupla resistência, no qual considera-se o efeito das resistências difusionais na superfície e no poro do adsorvente.

\section{MATERIAIS, MÉTODOS E MODELO}

Materiais: Os adsorventes mesoporosos MCM-41 foram sintetizados pelo Laboratório de Catálise da Universidade Federal do Rio Grande do Norte, com caracterização textural reportada em Luna et al. (2011). Como solvente utilizou-se o iso-octano (Sigma, pureza > 99\%, Brasil) e como HPA de referência foi utilizado pireno (Acros Organics, pureza > 98\%, EUA).

Experimentos em batelada: Foram verificadas recomendações da IUPAC para os ensaios de imersão. Estudos de equilíbrio termodinâmico com as misturas sintéticas foram realizados variando-se a concentração do adsorbato no fluido e mantendo-se constante a massa de adsorvente para as temperaturas de 30,45 e $60^{\circ} \mathrm{C}$. Por um balanço de massa, a concentração na fase sólida pode ser calculada pela Equação 1, sendo expressa em (mg) de aromáticos/(gads) de adsorvente.

$$
q^{*}=\frac{M_{s, i} C_{i}-M_{s, f} C_{f}}{M_{a d s}}
$$

Em que: $C_{i}$ é a concentração inicial de aromáticos $(\mathrm{mg} / \mathrm{g}) ; C_{f}$ é a concentração final de aromáticos $(\mathrm{mg} / \mathrm{g}) ; M_{s, i}$ é a massa da solução sintética inicial $(\mathrm{g}) ; M_{s, f}$ é a massa da solução sintética final $(\mathrm{g})$; $M_{a d s}$ é a massa de adsorvente ( $\left.\mathrm{g}_{\mathrm{ads}}\right)$; e $q^{*}$ é a concentração de adsorbato na fase sólida $\left(\mathrm{mg} / \mathrm{g}_{\text {ads }}\right)$. 
Os resultados dos experimentos de equilíbrio dos sistemas estudados foram tratados através das equações de Langmuir e Toth (Equação 2).

$$
\frac{q^{*}}{q_{m}}=\frac{k C_{e q}}{1+k C_{e q}} \text { (Langmuir) } \quad \frac{q^{*}}{q_{m}}=\frac{C_{e q}}{\left(b+C_{e q}{ }^{v}\right)^{1 / v}} \text { (Toth) }
$$

Em que: $q^{*}$ é a concentração de aromáticos na fase sólida $\left(\mathrm{mg} / \mathrm{g}_{\mathrm{ads}}\right) ; C_{e q}$ é a concentração de adsorbato no fluido em equilíbrio ( $\mathrm{mg} / \mathrm{g}) ; k$ é a constante de Langmuir $(\mathrm{g} / \mathrm{mg}) ; q_{m}$ é a capacidade máxima de adsorção $\left(\mathrm{mg} / \mathrm{g}_{\text {ads }}\right) ; b$ é um parâmetro relacionado com a afinidade com o adsorvente $\left(\mathrm{mg}^{v} / \mathrm{g}^{v}\right)$; e v é um parâmetro que está relacionado com o grau de heterogeneidade da superfície.

Experimentos em leito fixo: Uma coluna preenchida com o adsorvente foi conectada ao sistema de bombeamento do HPLC (Varian ProStar 210) conforme ilustrado na Figura 1.

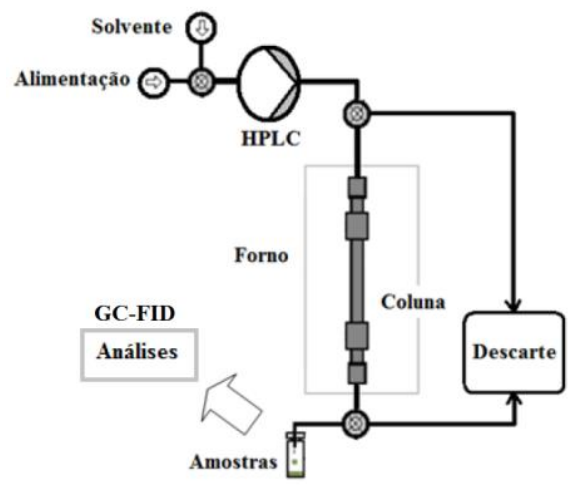

Figura 1 - Diagrama esquemático do sistema utilizado para a adsorção em leito fixo.

Inicialmente, foi injetado apenas solvente puro à coluna de modo a estabelecer a vazão desejada. Após esse estágio inicial, amostras do produto de saída eram coletadas e analisadas periodicamente, enquanto a corrente de entrada era injetada à coluna com concentrações crescentes de soluto $(50$ a $1000 \mathrm{ppm})$ e a $30^{\circ} \mathrm{C}$. A concentração de pireno foi analisada através dos equipamentos Varian CP-3800, acoplado a coluna capilar CP-Sil $8 \mathrm{CB}$ de $30 \mathrm{~m}$ de comprimento, $0.25 \mathrm{~mm}$ de diâmetro e $0.2 \mu \mathrm{m}$ de espessura de filme, e detector de ionização de chamas (FID).

Os experimentos com passos sucessivos foram realizados de acordo com o método aplicado por Sajonz (1996), no qual não há eluição entre as injeções de correntes de concentrações subsequentes. Para o cálculo da quantidade adsorvida em equilíbrio com a concentração de alimentação foi necessário somar a quantidade do estágio anterior, conforme a Equação 3.

$$
q_{n+1}=q_{n}+\frac{C_{n+1}}{M_{L}}\left[Q \int_{t(n)}^{t(n+1)}\left(1-\frac{C}{C_{n+1}}\right) d t-V_{L} \cdot \varepsilon\right]
$$


Em que: $q_{n}$ e $q_{n+1}$ são as quantidades adsorvidas pela fase sólida após o n-ésimo e o (n-ésimo +1$)$ passos $\left(\mathrm{mg} / \mathrm{g}_{\mathrm{ads}}\right)$, quando em equilíbrio com a concentração $C_{n+1}(\mathrm{mg} / \mathrm{L}) ; M_{L}$ é a massa de adsorvente no leito ( $\left.\mathrm{g}_{\mathrm{ads}}\right)$; Q é a vazão volumétrica ( $\left.\mathrm{L} / \mathrm{min}\right)$; C é a concentração de saída $(\mathrm{mg} / \mathrm{L})$; $V_{L}$ é o volume do leito (L); e $\varepsilon$ é a porosidade do leito.

Simulação das curvas de ruptura: Para análise das curvas de ruptura, o modelo geral de transferência de massa de dupla resistência intrapartícula foi utilizado. A transferência de massa do seio do fluido ao interior da partícula passa por três resistências, resultantes da difusão pelo filme externo, difusão nos poros e a difusão na superfície das partículas, gerando gradiente de concentração na direção radial do adsorvente. Assumiu-se também que a adsorção ocorre instantaneamente, uma vez que a taxa à qual ocorre é desprezível em relação às outras etapas.

O modelo estima a difusividade na superfície do poro, $D_{S}$, através dos resultados obtidos experimentalmente e de valores encontrados na literatura para o coeficiente de transferência de massa no filme $(\mathrm{cm} / \mathrm{min})$, a dispersão axial $\left(\mathrm{cm}^{2} / \mathrm{min}\right)$ e a difusividade no poro $\left(\mathrm{cm}^{2} / \mathrm{min}\right)-k_{f}, D_{L}$ e $D_{P}$, respectivamente - conforme reportado por Luna et al. (2011). O modelo é completamente descrito pelas Equações 4 a 10.

Balanço de massa diferencial na fase líquida:

$$
\varepsilon \frac{\partial C}{\partial t}+u \frac{\partial C}{\partial z}=\varepsilon D_{L} \frac{\partial^{2} C}{\partial z^{2}}-(1-\varepsilon) \frac{3 k_{f}}{R}\left(C-\left.C_{p}\right|_{r=R_{p}}\right)
$$

Condições iniciais:

$$
t=0, \quad C(z, 0)=C_{n-1}, \quad q(z, 0)=q_{n-1}
$$

Condições de contorno para fase líquida:

$$
\begin{array}{ll}
z=0, & u C(0, t)-D_{L} \frac{\partial C}{\partial z}=u\left(C_{n}-C_{n-1}\right) \\
z=L, & \frac{\partial C(L, t)}{\partial z}=0
\end{array}
$$

Balanço diferencial para fase sólida:

$$
\varepsilon_{p} \frac{\partial C_{p}}{\partial t}+\left(1-\varepsilon_{p}\right) \frac{\partial q}{\partial t} \rho_{a p}=D_{p} \frac{1}{r^{2}} \frac{\partial}{\partial r}\left(r^{2} \frac{\partial C_{p}}{\partial r}\right)+D_{S} \frac{1}{r^{2}}\left(r^{2} \frac{\partial q}{\partial r}\right)
$$


Condições de contorno para a fase sólida:

$$
\begin{aligned}
& r=0, \quad \frac{\partial C_{p}}{\partial r}=0 \\
& r=R_{p}, \quad D_{p} \frac{\partial C_{p}}{\partial r}+D_{S} \frac{\partial q}{\partial r} \rho_{a p}=k_{f}\left(C-C_{p}\right)
\end{aligned}
$$

Em que: $C_{p}$ é a concentração da fase líquida intraparticular $(\mathrm{mg} / \mathrm{L})$ e $q$ é a concentração na fase adsorvida $\left(\mathrm{mg} / \mathrm{g}_{\mathrm{ads}}\right)$, que podem ser relacionadas através da equação de Langmuir; $C$ é a concentração na fase líquida escoando no leito $(\mathrm{mg} / \mathrm{L}) ; u$ é a velocidade superficial $(\mathrm{cm} / \mathrm{min}) ; \rho_{\mathrm{ap}}$ é a densidade aparente do adsorvente ( $\left.\mathrm{g}_{\text {ads }} / \mathrm{L}\right)$; e $\varepsilon_{\mathrm{p}}$ é a porosidade do adsorvente.

Procedimentos numéricos: O simulador comercial gPROMS foi utilizado para solucionar numericamente o modelo supracitado que consistiu em um sistema de equações diferenciais parciais, incluindo equações algébricas. Os domínios axial e radial foram discretizados usando o método de colocação ortogonal de terceira ordem em elementos finitos (OCFEM). Os parâmetros de transferência de massa no sistema foram estimados através de um pacote de otimização, usando o método heterocedástico, versão 3.1 (gPROMS User Guide, 2006).

\section{RESULTADOS E DISCUSSÃO}

Experimentos em batelada: As isotermas foram construídas para o material MCM-41, e a capacidade máxima de adsorção de pireno foi estimada a partir das equações de Langmuir e Toth, cujo valor para temperatura de $30^{\circ} \mathrm{C}$ foi de $0,804 \mathrm{mmol} / \mathrm{g}$, segundo a equação de Toth. A adsorção do solvente foi considerada negligenciável para os cálculos de balanço de massa. As curvas obtidas estão apresentadas na Figura 3.

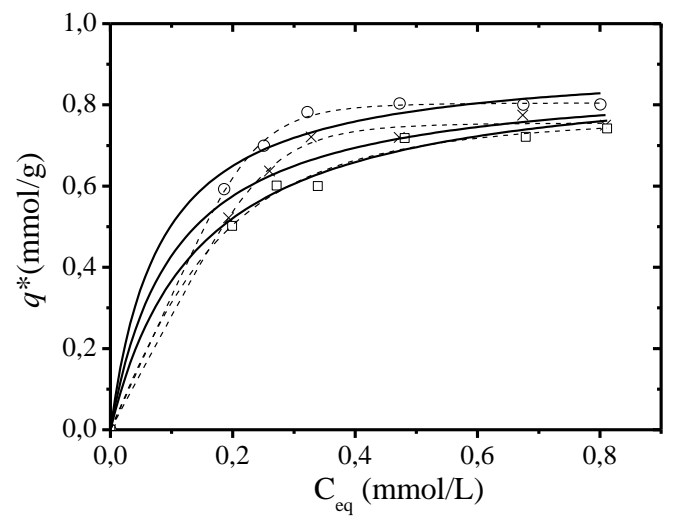

Figura 3 - Isotermas de adsorção de pireno sobre MCM-41 a diferentes temperaturas (口) $30{ }^{\circ} \mathrm{C}$; (×) $45^{\circ} \mathrm{C}$; (O) $60^{\circ} \mathrm{C}$; Ajuste utilizando a equação de Langmuir (-) e Toth (- -). 
De acordo com os resultados, as equações de Langmuir e Toth foram adequadas para representar a adsorção de pireno com coeficientes de correlação $\left(\mathrm{R}^{2}\right)$ entre 0,95 e 0,99 . Estes resultados indicaram uma boa associação entre os valores experimentais e os preditos com erros médios inferiores a 5\%.

Experimentos em leito fixo: A dinâmica do processo de adsorção bem como a estimativa dos parâmetros de transferência de massa (Tabela 2) foram estudadas para o adsorvente MCM-41 a temperatura de $30^{\circ} \mathrm{C}$, com concentrações de misturas sintéticas de 50 a $1000 \mathrm{mg} / \mathrm{kg}$. As curvas de ruptura construídas estão apresentadas na Figura 4 apenas para a menor e maior concentração de alimentação, em que pode ser observado uma boa concordância entre os dados experimentais e os simulados.

Tabela 2 - Parâmetros do modelo e difusividade superficial estimada para o sistema de pireno em iso-octano sobre $\mathrm{MCM}-41$ a $30^{\circ} \mathrm{C}$ para diferentes concentrações de alimentação.

\begin{tabular}{|c|c|c|c|}
\hline$\overline{C_{0}(\mathrm{mmol} / \mathrm{L})}$ & $D_{L}\left(\mathrm{~cm}^{2} / \mathrm{min}\right)$ & $k_{f}(\mathrm{~cm} / \mathrm{min})$ & $D_{S}\left(\mathrm{~cm}^{2} / \mathrm{min}\right)$ \\
\hline 0,17 & \multirow{5}{*}{2,60} & \multirow{5}{*}{2,21} & $2,05 \cdot 10^{-10}$ \\
\hline 0,37 & & & $2,79 \cdot 10^{-10}$ \\
\hline 0,71 & & & $1,01 \cdot 10^{-9}$ \\
\hline 1,76 & & & $1,92 \cdot 10^{-9}$ \\
\hline 3,57 & & & $2,33 \cdot 10^{-9}$ \\
\hline
\end{tabular}

Para afirmar que o modelo utilizado gerou uma boa previsão dos perfis de concentração de saída do leito foi utilizada a análise de variância (ANOVA) para verificar as diferenças entre os conjuntos de dados experimentais e as respectivas simulações, com um nível de significância de 0,05. Os parâmetros $p$, que estão relacionados com a confiabilidade das simulações, foram sempre maiores que 0,98 para as curvas de ruptura com misturas modelo.
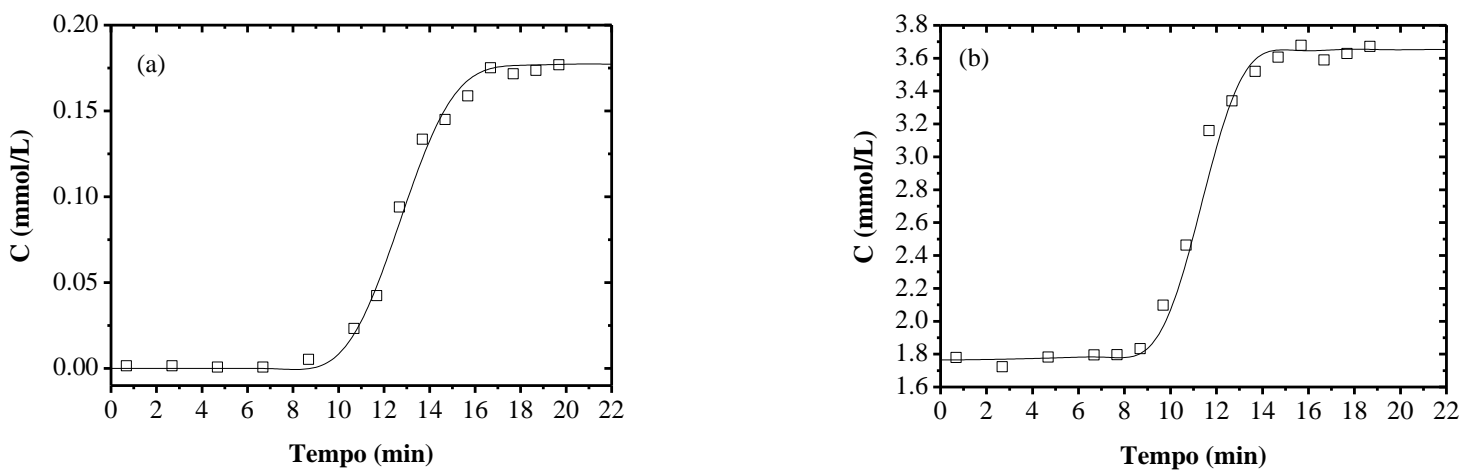

Figura 4 - Curvas de ruptura de pireno sobre MCM-41 a $30^{\circ} \mathrm{C}$; (a) $\mathrm{C}_{0}=0,190 \mathrm{mmol} / \mathrm{L}$ (50 mg/kg); (b) $\mathrm{C}_{0}=3,502 \mathrm{mmol} / \mathrm{L}(1.000 \mathrm{mg} / \mathrm{kg}) ; \mathrm{Q}=0,20 \mathrm{~mL} / \mathrm{min}$. 
A partir do balanço de massa integral ao longo do tempo, foi visto também que as capacidades de adsorção estão coerentes com os valores estimados utilizando os experimentos em batelada. Os valores de $D s$ estimados para a adsorção de pireno, mostraram uma dependência com a concentração de alimentação. Foi observado que $D s$ aumentou com o aumento da concentração de entrada, conforme reportado por Luna et al. (2011) para carbonos ativados mesoporosos.

\section{CONCLUSÃO}

Neste trabalho foi estudado o processo de adsorção de um poliaromático padrão (pireno) visando a determinação das propriedades fundamentais de equilíbrio e de transferência de massa deste composto, em mistura de hidrocarbonetos, sobre materiais mesoporosos do tipo MCM-41. Os experimentos realizados serviram para avaliação dos materiais e determinação dos parâmetros de transferência de massas que são importantes para aplicação e ampliação de escala.

Os materiais estudados têm potencial para remoção de aromáticos em correntes de hidrocarbonetos, pois apresentaram elevada capacidade de adsorção de HPAs (184 mg/g). Para as isotermas obtidas os valores de $q_{m}$ diminuíram com o aumento da temperatura, o que confirmou o caráter exotérmico do fenômeno de fisissorção.

A dinâmica de adsorção em leito fixo foi utilizada para avaliar e confirmar a elevada capacidade dos materiais e também para estimar a difusividade superficial efetiva no interior das partículas. Os valores obtidos para as difusividades apresentam coerentes a outros resultados para materiais mesoporosos.

Através dos resultados pode ser observado que o modelo utilizado conseguiu predizer o comportamento ruptura, estimar e confirmar os parâmetros de transferência de massa, calculados a partir de correlações, representando com confiabilidade o processo de adsorção de poliaromáticos em MCM-41 de forma contínua.

\section{AGRADECIMENTOS}

Os autores agradecem ao Conselho Nacional de Desenvolvimento Científico e Tecnológico $(\mathrm{CNPq})$ e a Petrobras pelo apoio financeiro e logístico.

\section{REFERÊNCIAS}

Agency for toxic substances disease registry (ATSDR). Toxicological profile for polycyclic aromatic hydrocarbons (PAHs). Atlanta: U.S. Department of Health and Human Services, Public Health Service, 1995.

Ania, C. O.; Cabal, B.; Pevida, C.; Arenillas, A.; Parra, J. B.; Rubiera, F.; Pis, J. J. Removal of Naphthalene from Aqueous Solution on Chemically Modified Activated Carbons. Water Res., v. 41, p. 333, 2007. 


\section{9 a 22 de outubro de 2014 \\ Florianópolis/SC}

Araújo, R. S.; Azevedo, D.C.S.; Cavalcante Jr., C.L.; Jiménez-López, A.; Rodríguez-Castellón, E. Adsorption of polycyclic aromatic hydrocarbons (PAHs) from isoctane solutions by mesoporous molecular sieves: Influence of the surface acidity. Microporous and Mesoporous Materials, v. 108, p. 213-222, 2008.

Do, D. D.; Rice, R. G.; On the relative importance of pore and surface diffusion in nonequilibrium adsorption rate processes. Chemical Engineering Science, v. 42, p. 2269-2284, 1987.

Gan, S.; Lau, E.V.; Ng, H. K. Remediation of soils contaminated with polycyclic aromatic hydrocarbons (PAHs). Journal of Hazardous Materials, v. 172, p. 532-549, 2009.

Ghiaci, M.; Abbaspur, A.; Kia, R.; Seyedeyn-Azad, F. Equilibrium isotherm studies for the sorption of benzene, toluene, and phenol onto organo-zeolites and as-synthesized MCM-41, Separation and Purification Technology, v. 40, p. 217-229, 2004.

gPROMS, "User Guide", Advanced process modeling and simulation, Process System Enterprise, 2006.

Juang, L-C.; Wang, C-C.; Lee, C-K. Adsorption of basic dyes onto MCM-41. Chemosphere, v. 64, p. 1920-1928, 2006.

Luna, F.M.T.; Araujo, C.C.B.; Veloso, C.B.; Silva Jr., I.J.; Azevedo, D.C.S.; Cavalcante Jr., C.L. Adsorption of Naphthalene and Pyrene on Commercial Activated Carbons. Adsorption. v. 17, p. 937-947, 2011.

Luna, F.M.T.; Pontes-Filho, A.A.; Trindade, E.D.; Silva Jr., I.J.; Azevedo, D.C.S.; Cavalcante Jr., C.L. Removal of Aromatic Compounds from Mineral Naphthenic Oil by Adsorption. Industrial \& Engineering Chemistry Research, v. 14, p. 72-78, 2008.

Mastral, A. M.; García, T.; Callén, M. S.; Murillo, R.; Navarro, M. V. ; López, J. M. Sorbent characteristics influence on the adsorption of PAC: I. PAH adsorption with the same number of rings. Fuel Processing Technology, v. 77-78, p. 373-379, 2002.

Northcotta, K. A.; Miyakawa, K.; Oshima, S; Komatsu, Y.; Perera, J.M.; Stevens, G.W. The adsorption of divalent metal cations on mesoporous silicate MCM-41. Chemical Engineering Journal, v. 157, p. 25-28, 2010.

Puanngama, M.; Unob, F. Preparation and use of chemically modified MCM-41 and silica gel as selective adsorbents for $\mathrm{Hg}$ (II) ions. Journal of Hazardous Materials, v. 154, p. 578-587, 2008.

Sajonz, P.; Zhong, G.; Guiochon, G.; Influence of the concentration dependence of the mass transfer properties on chromatographic band profiles II. Accuracy of the determination of isotherm data by frontal analysis, Journal of Chromatography A, v. 731, p. 1-25, 1996.

Wang, L. C.; Wang, I. C.; Chang, J. E.; Lai, S.O.; Chien, G.P.C. Emission of polycyclicaromatic hydrocarbons (PAHs) from the liquid injection incineration of petrochemical industrial wastewater. J. Hazardous Material , v. 148, p. 296-302, 2007. 